Supplementary material

\title{
Assessing Future Habitat Availability for Coastal Lowland Anurans in the Brazilian Atlantic Rainforest
}

Igor Soares de Oliveira ${ }^{1,2, *}$, Dennis Rödder ${ }^{3}$, César Capinha $^{4}$, Faraham Ahmadzadeh $^{3}$, Adriele Karlokoski Cunha de Oliveira ${ }^{5}$, and Luís Felipe Toledo $^{2}$

${ }^{1}$ Programa de Pós-Graduação em Ecologia, Instituto de Biologia, Universidade Estadual de Campinas, Avenida Bertrand Russel, s/n, Cidade Universitária Zeferino Vaz, Barão Geraldo, CEP 13083-865, Campinas, São Paulo, Brasil

${ }^{2}$ Laboratório de História Natural de Anfíbios Brasileiros, Departamento de Biologia Animal, Instituto de Biologia, Unicamp, Rua Monteiro Lobato, 255, CEP 13083-862, Campinas, São Paulo, Brasil

${ }^{3}$ Zoologisches Forschungsmuseum Alexander Koenig, Herpetology Section, Adenauerallee 160, 53113 Bonn, Germany.

${ }^{4}$ Centro de Biologia Ambiental, Faculdade de Ciências da Universidade de Lisboa, 1749-016 Lisboa, Portugal.

${ }^{5}$ Programa de Pós-Graduação em Zoologia, Departamento de Zoologia, Universidade Federal do Paraná, Rua Cel. Francisco Heráclito dos Santos, 210, Jardim das Américas, CEP 81531-970, Curitiba, Paraná, Brasil.

*Corresponding email address: oliveira.snake@ gmail.com Received: 


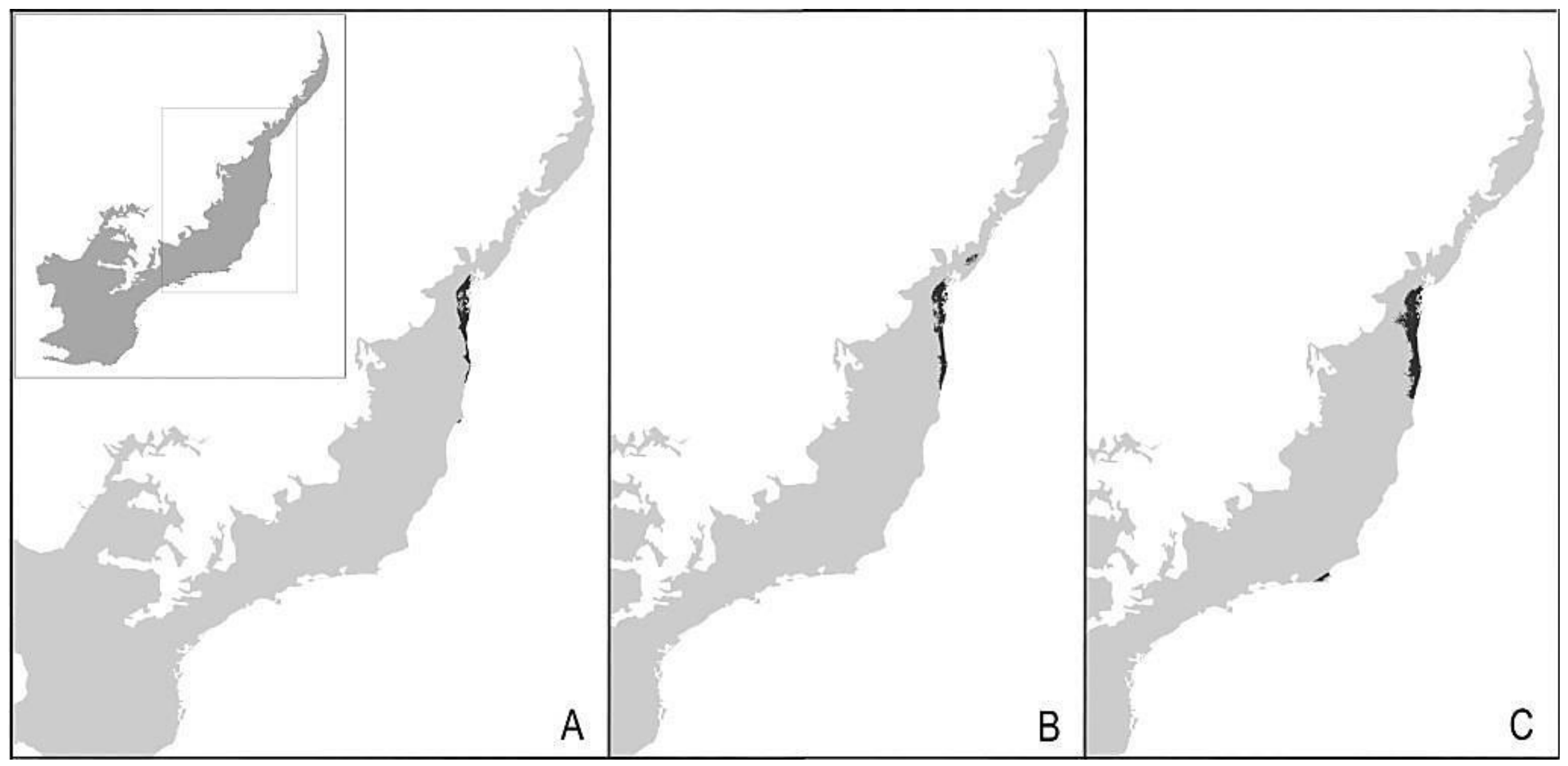

FIGURE S1: Estimated current (A) and future habitat suitability in the B2a (B) and A2a (C) climatic scenario for the year 2080 in the Brazilian Atlantic forest for Aparasphenodon arapapa. Modeled distributions shown in black. 


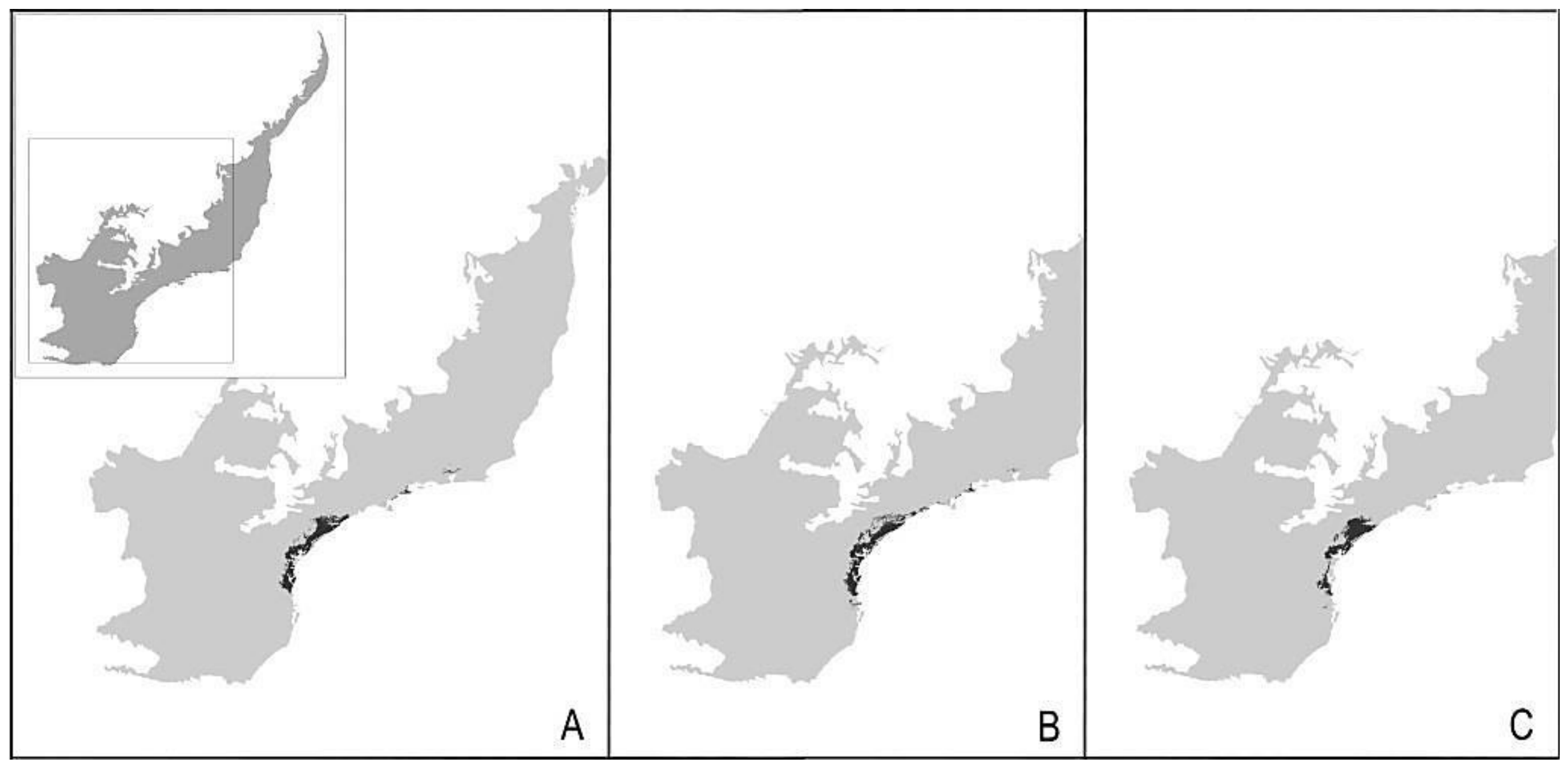

FIGURE S2: Estimated current (A) and future habitat suitability in the B2a (B) and A2a (C) climatic scenario for the year 2080 in the Brazilian Atlantic forest for A. bokermanni. Modeled distributions shown in black. 


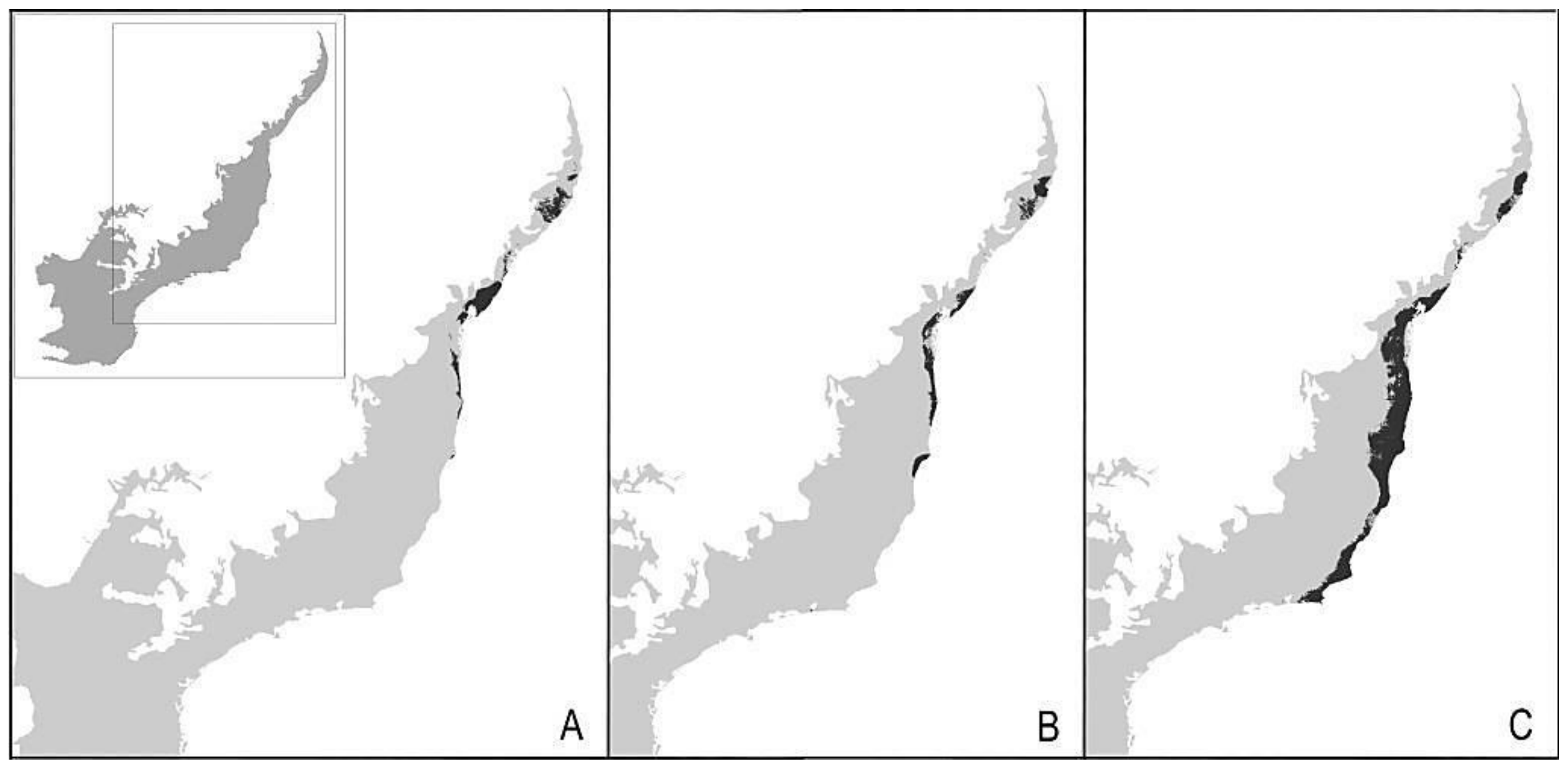

FIGURE S3: Estimated current (A) and future habitat suitability in the B2a (B) and A2a (C) climatic scenario for the year 2080 in the Brazilian Atlantic forest for Chiasmocleis alagoana. Modeled distributions shown in black. 


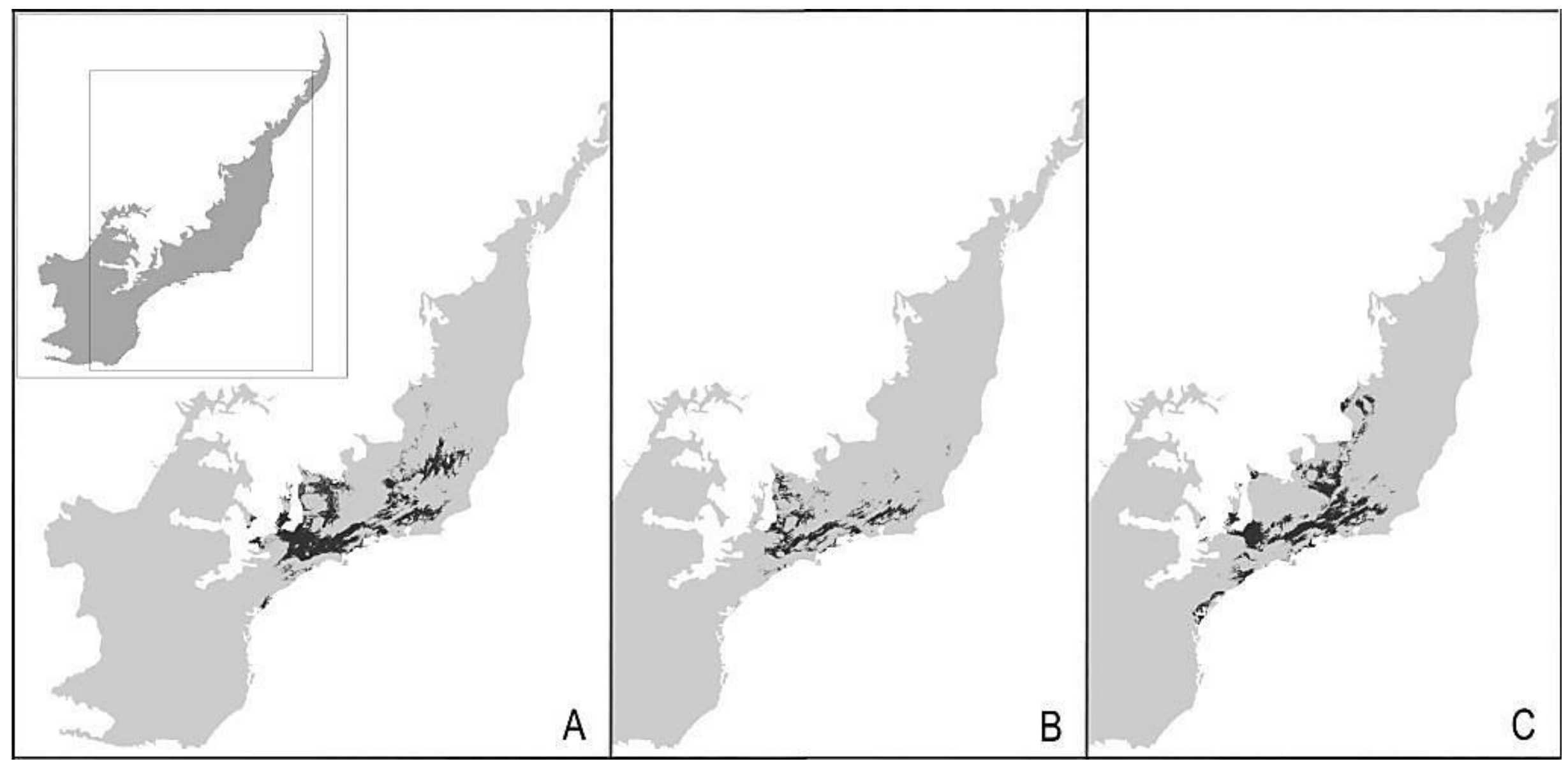

FIGURE S4: Estimated current (A) and future habitat suitability in the B2a (B) and A2a (C) climatic scenario for the year 2080 in the Brazilian Atlantic forest for C. atlantica. Modeled distributions shown in black. 


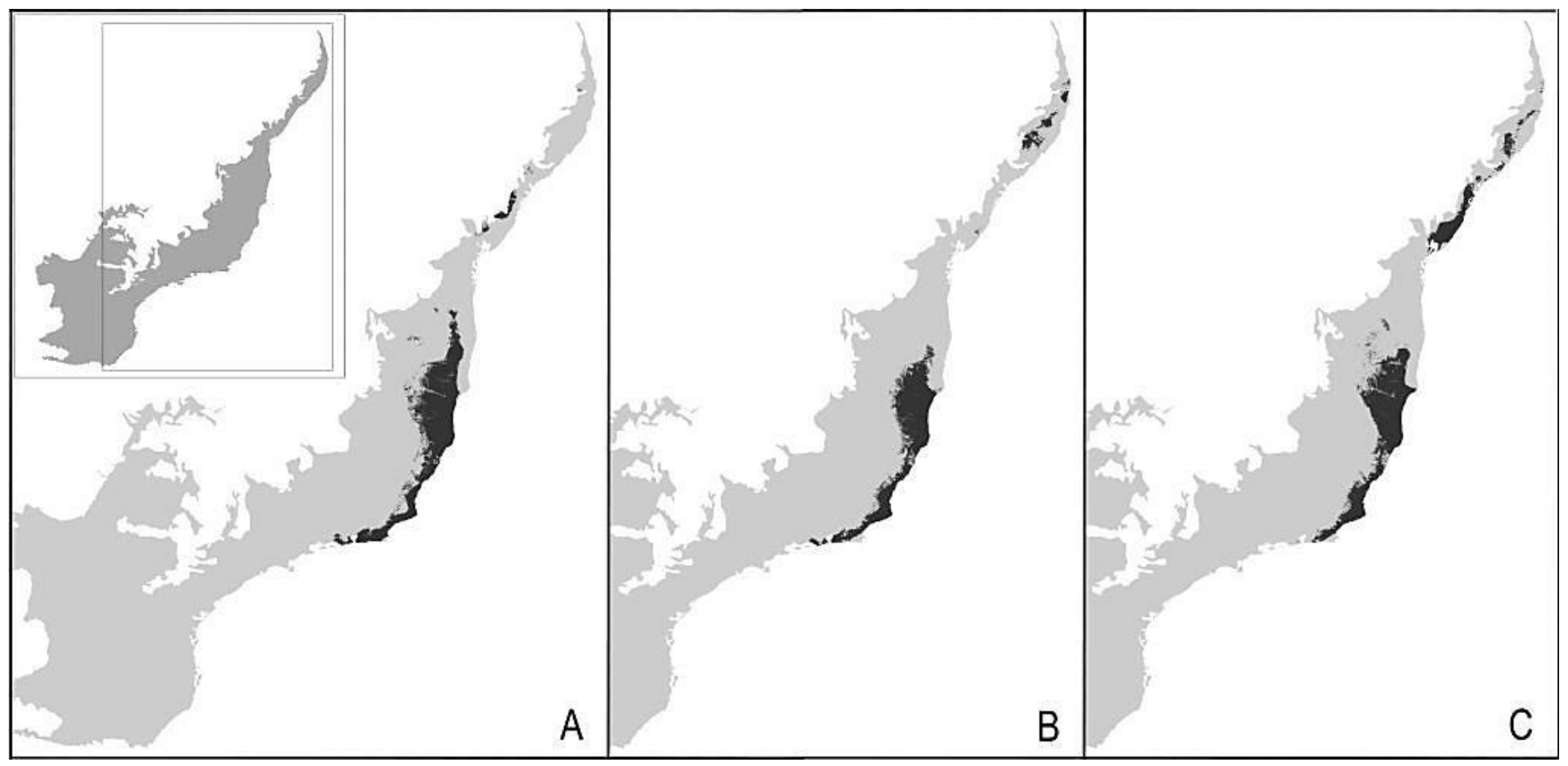

FIGURE S5: Estimated current (A) and future habitat suitability in the B2a (B) and A2a (C) climatic scenario for the year 2080 in the Brazilian Atlantic forest for $C$. capixaba. Modeled distributions shown in black. 


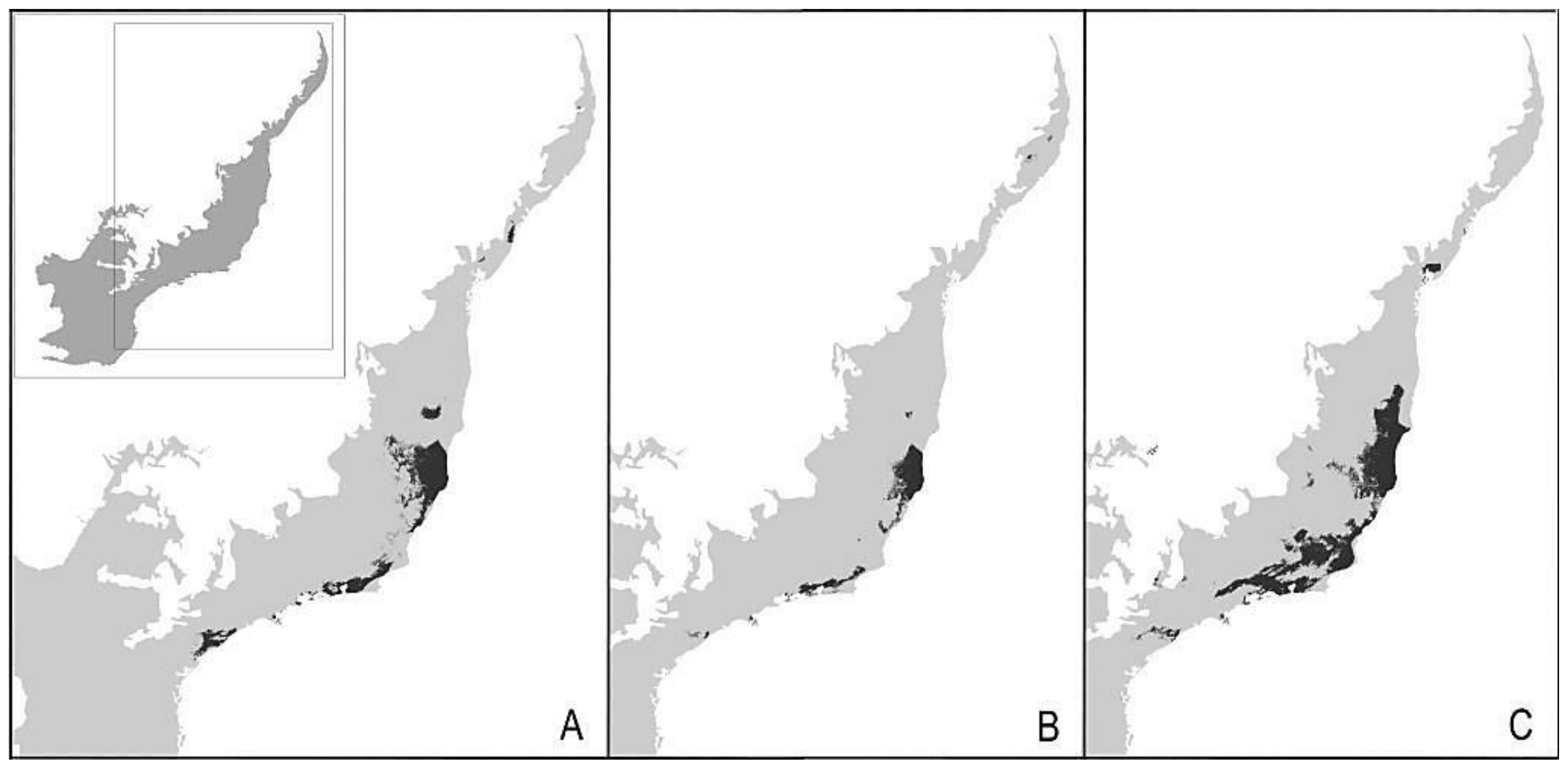

FIGURE S6: Estimated current (A) and future habitat suitability in the B2a (B) and A2a (C) climatic scenario for the year 2080 in the Brazilian Atlantic forest for C. lacrimae. Modeled distributions shown in black. 


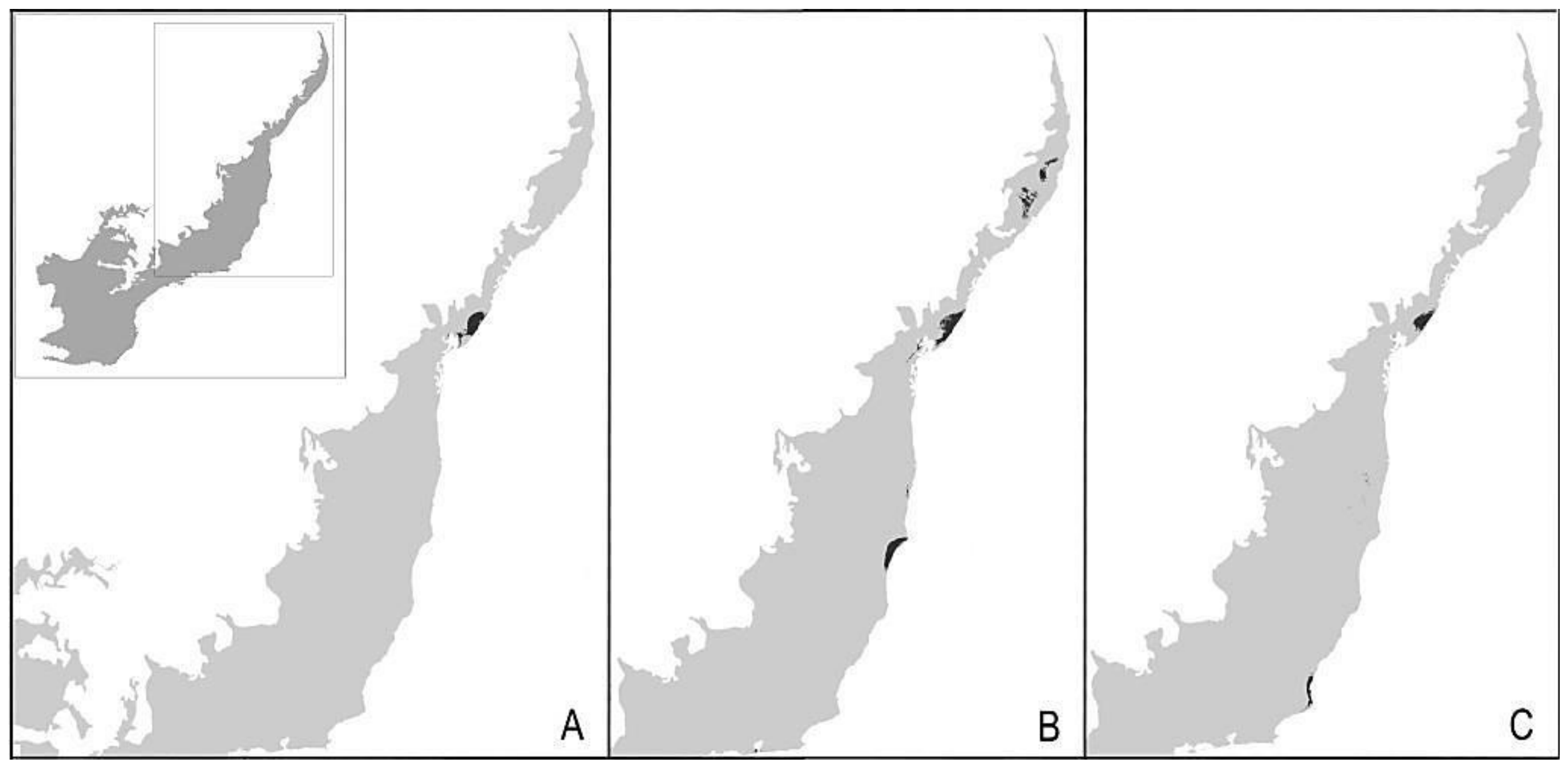

FIGURE S7: Estimated current (A) and future habitat suitability in the B2a (B) and A2a (C) climatic scenario for the year 2080 in the Brazilian Atlantic forest for C. sapiranga. Modeled distributions shown in black. 


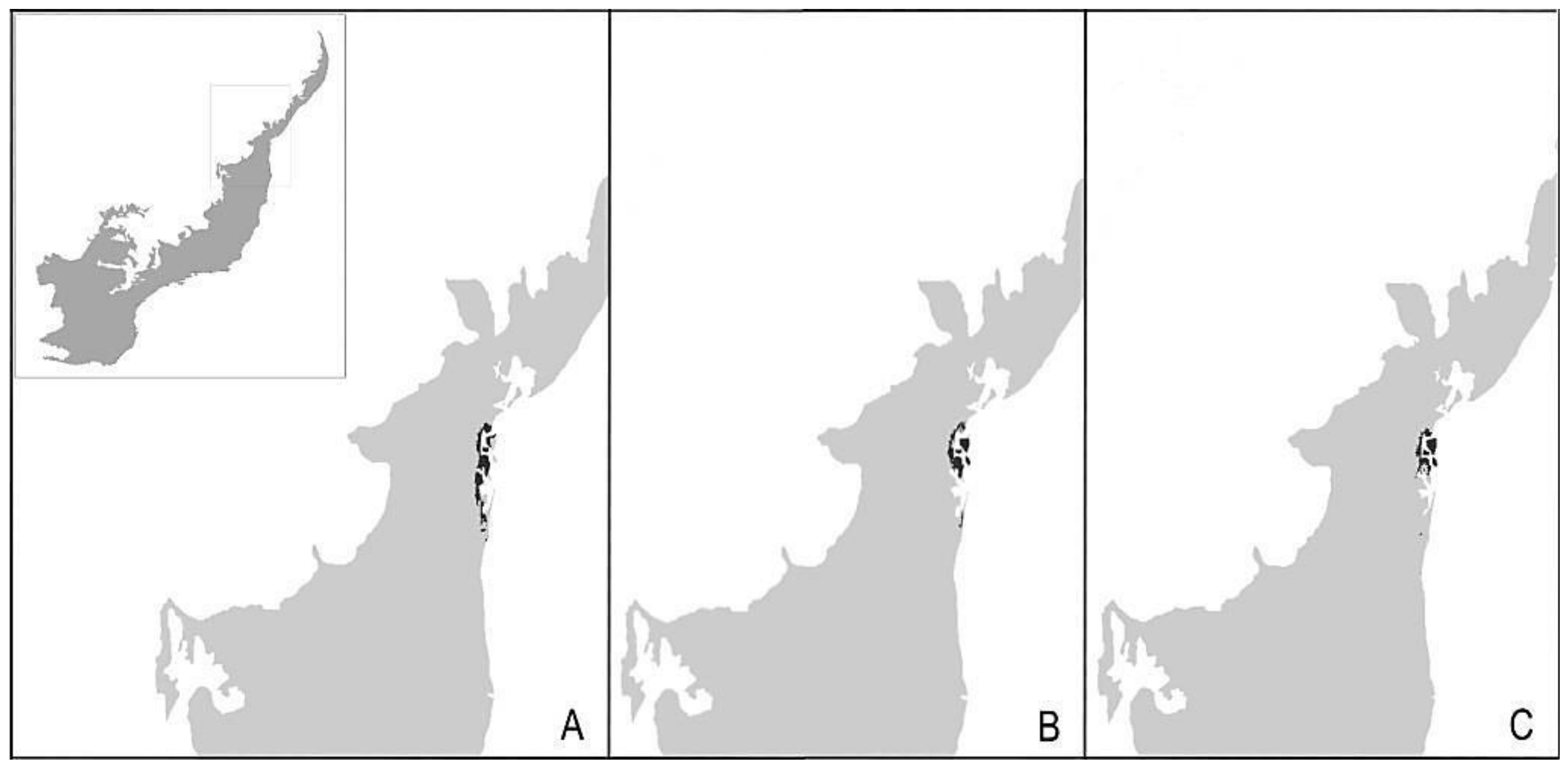

FIGURE S8: Estimated current (A) and future habitat suitability in the B2a (B) and A2a (C) climatic scenario for the year 2080 in the Brazilian Atlantic forest for Crossodactylus lutzorum. Modeled distributions shown in black. 


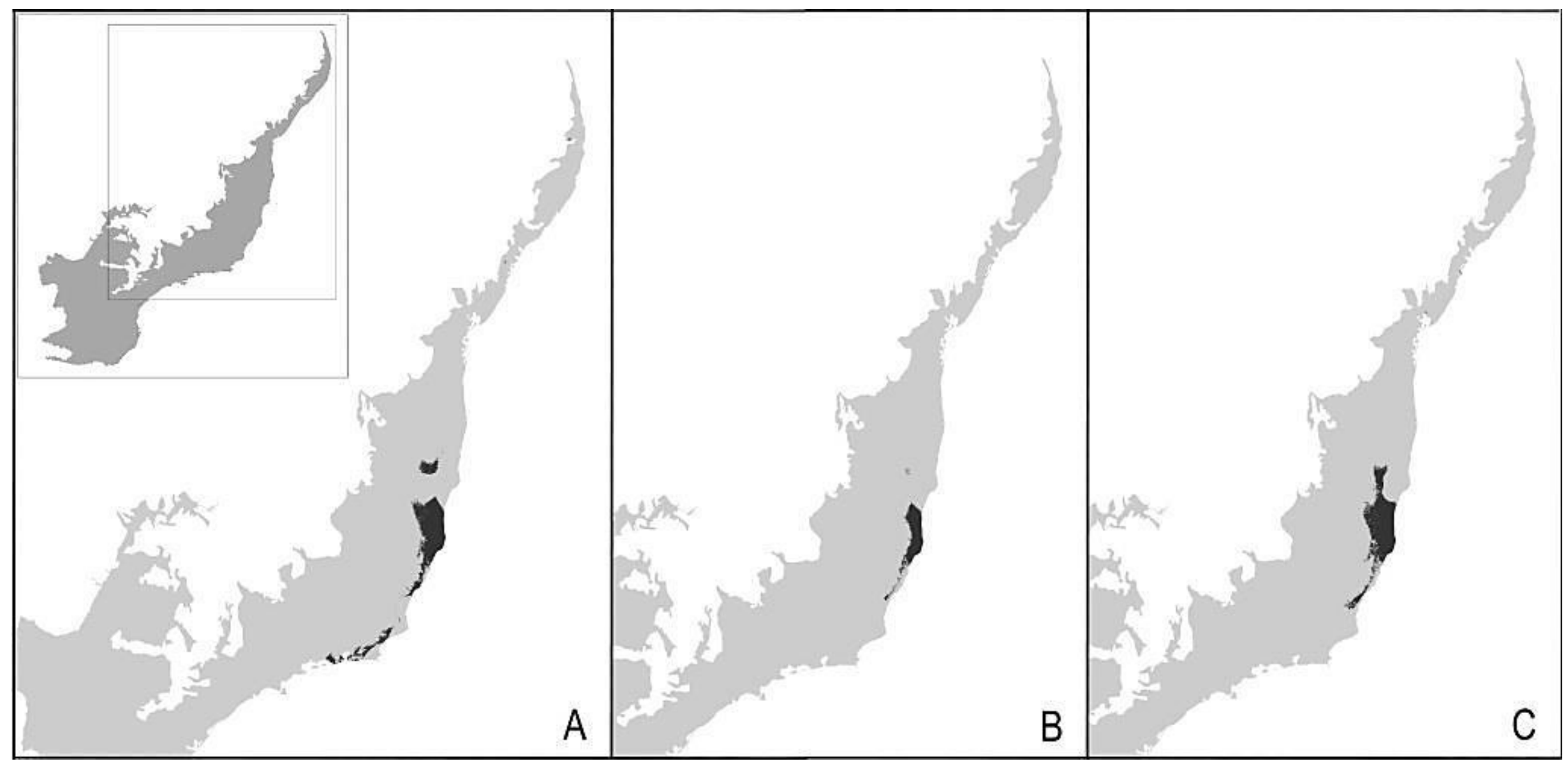

FIGURE S9: Estimated current (A) and future habitat suitability in the B2a (B) and A2a (C) climatic scenario for the year 2080 in the Brazilian Atlantic forest for Dasypops schirchi. Modeled distributions shown in black. 


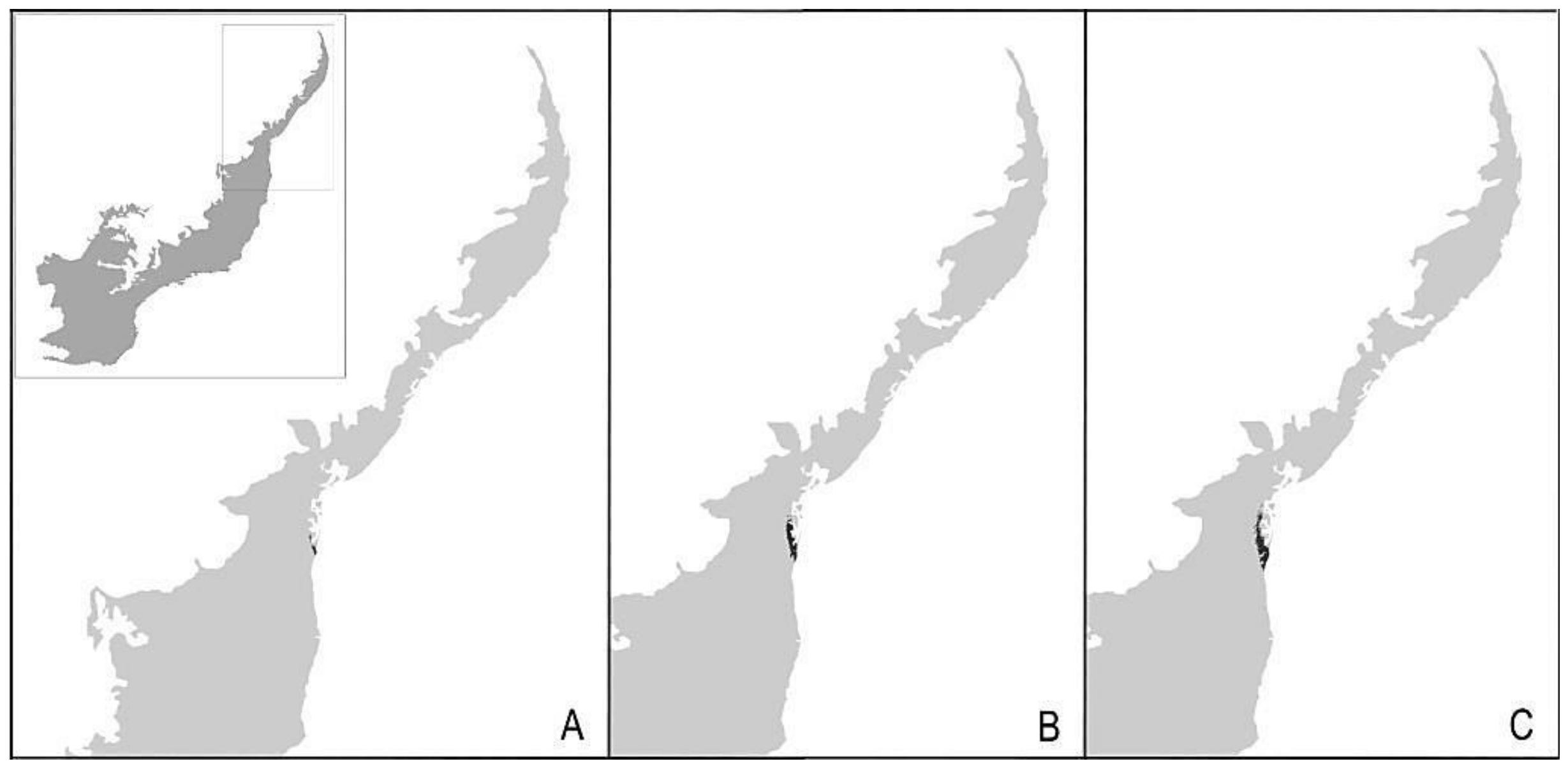

FIGURE S10: Estimated current (A) and future habitat suitability in the B2a (B) and A2a (C) climatic scenario for the year 2080 in the Brazilian Atlantic forest for Dendrophryniscus skuki. Modeled distributions shown in black. 


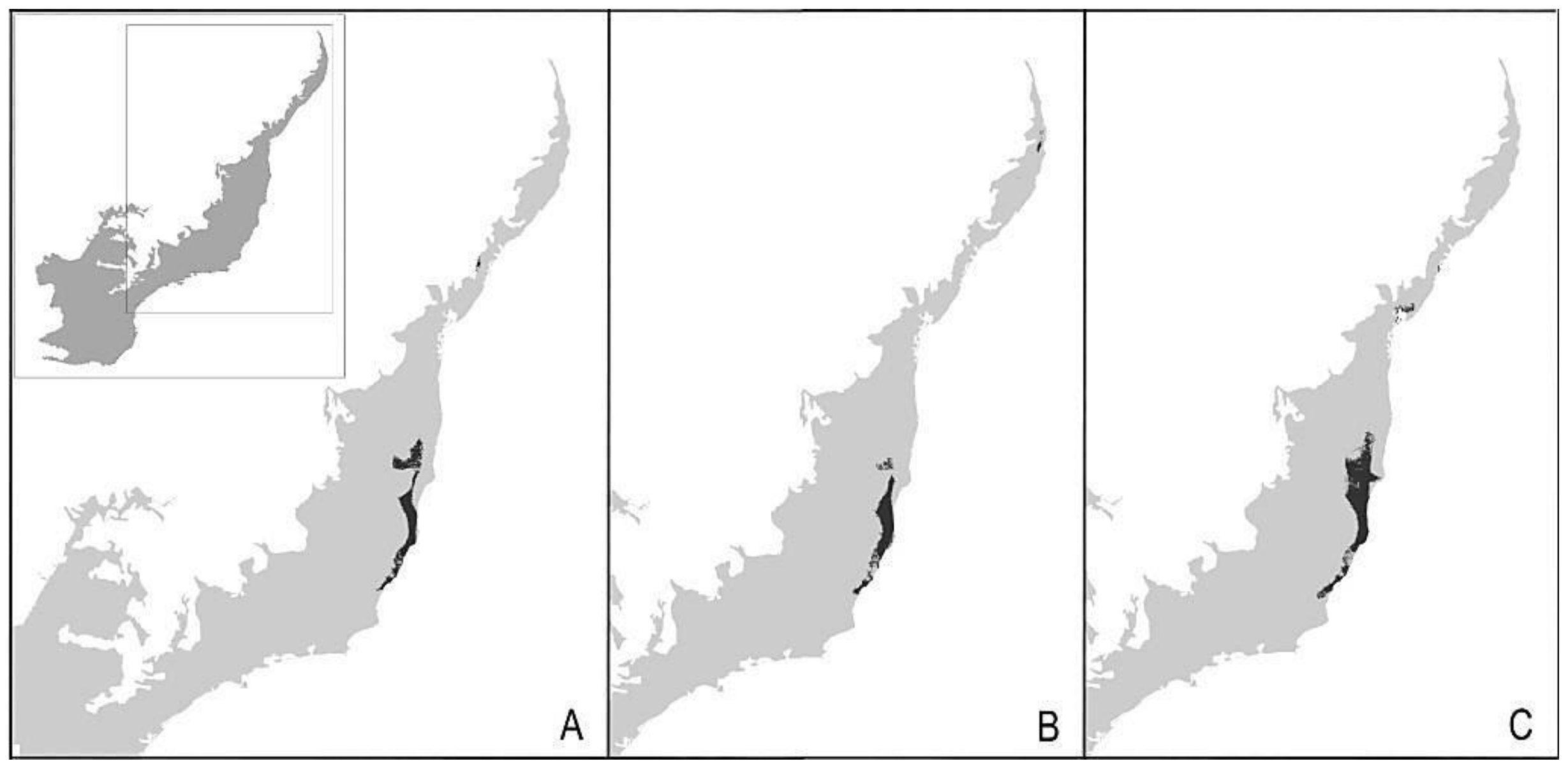

FIGURE S11: Estimated current (A) and future habitat suitability in the B2a (B) and A2a (C) climatic scenario for the year 2080 in the Brazilian Atlantic forest for Gastrotheca megacephala. Modeled distributions shown in black. 


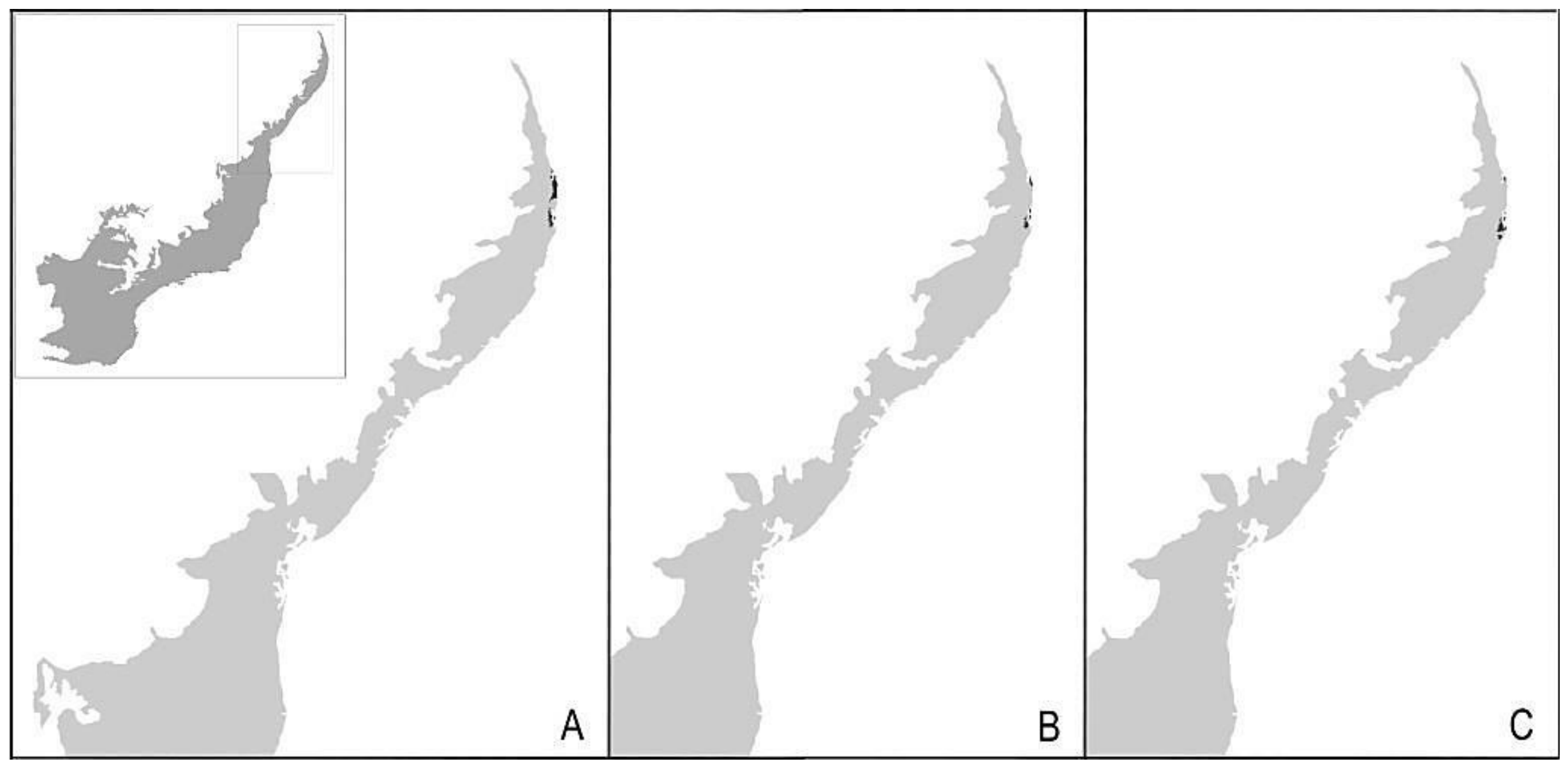

FIGURE S12: Estimated current (A) and future habitat suitability in the B2a (B) and A2a (C) climatic scenario for the year 2080 in the Brazilian Atlantic forest for Haddadus plicifer. Modeled distributions shown in black. 


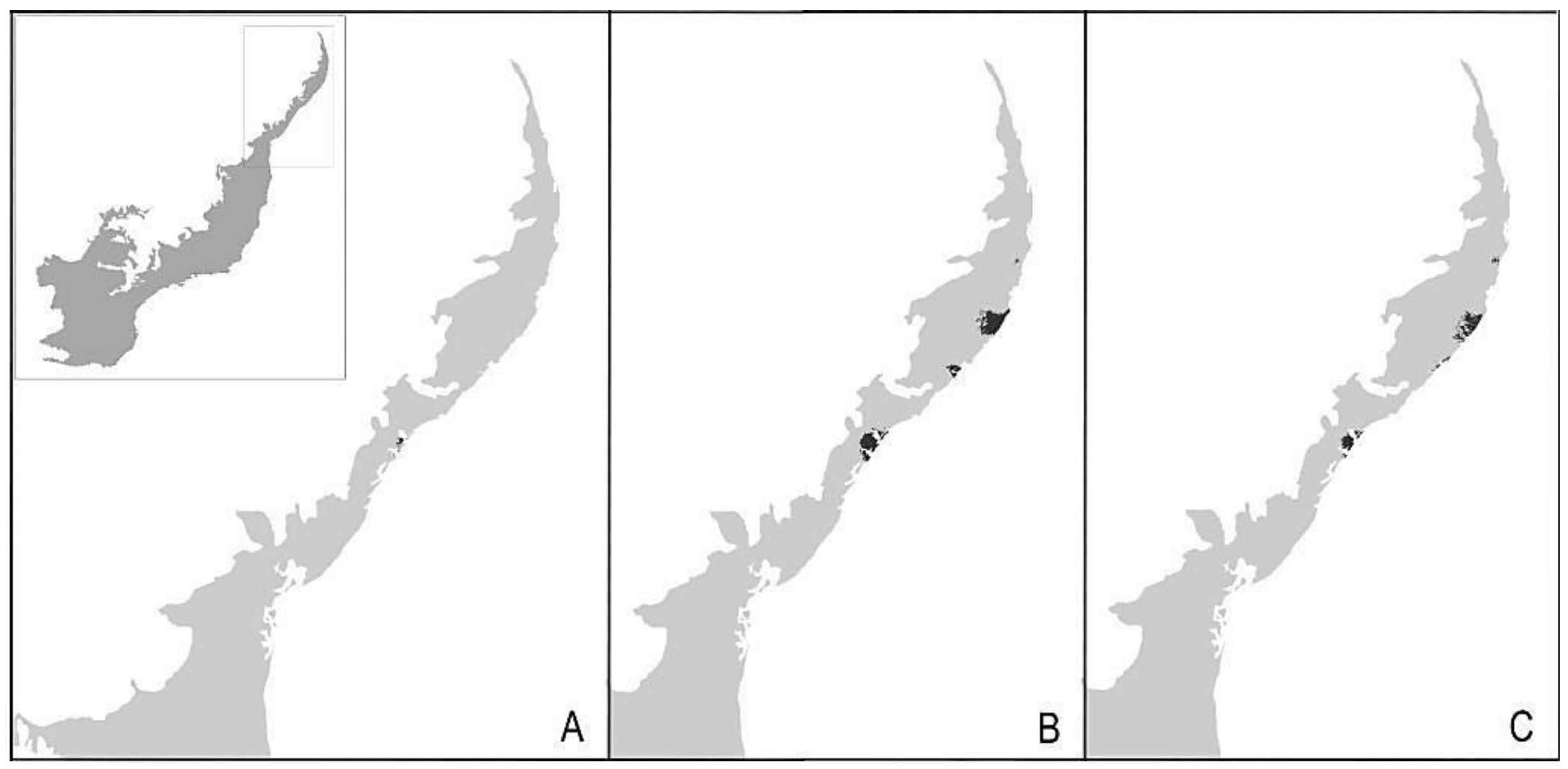

FIGURE S13: Estimated current (A) and future habitat suitability in the B2a (B) and A2a (C) climatic scenario for the year 2080 in the Brazilian Atlantic forest for Leptodactylus hylodes. Modeled distributions shown in black. 


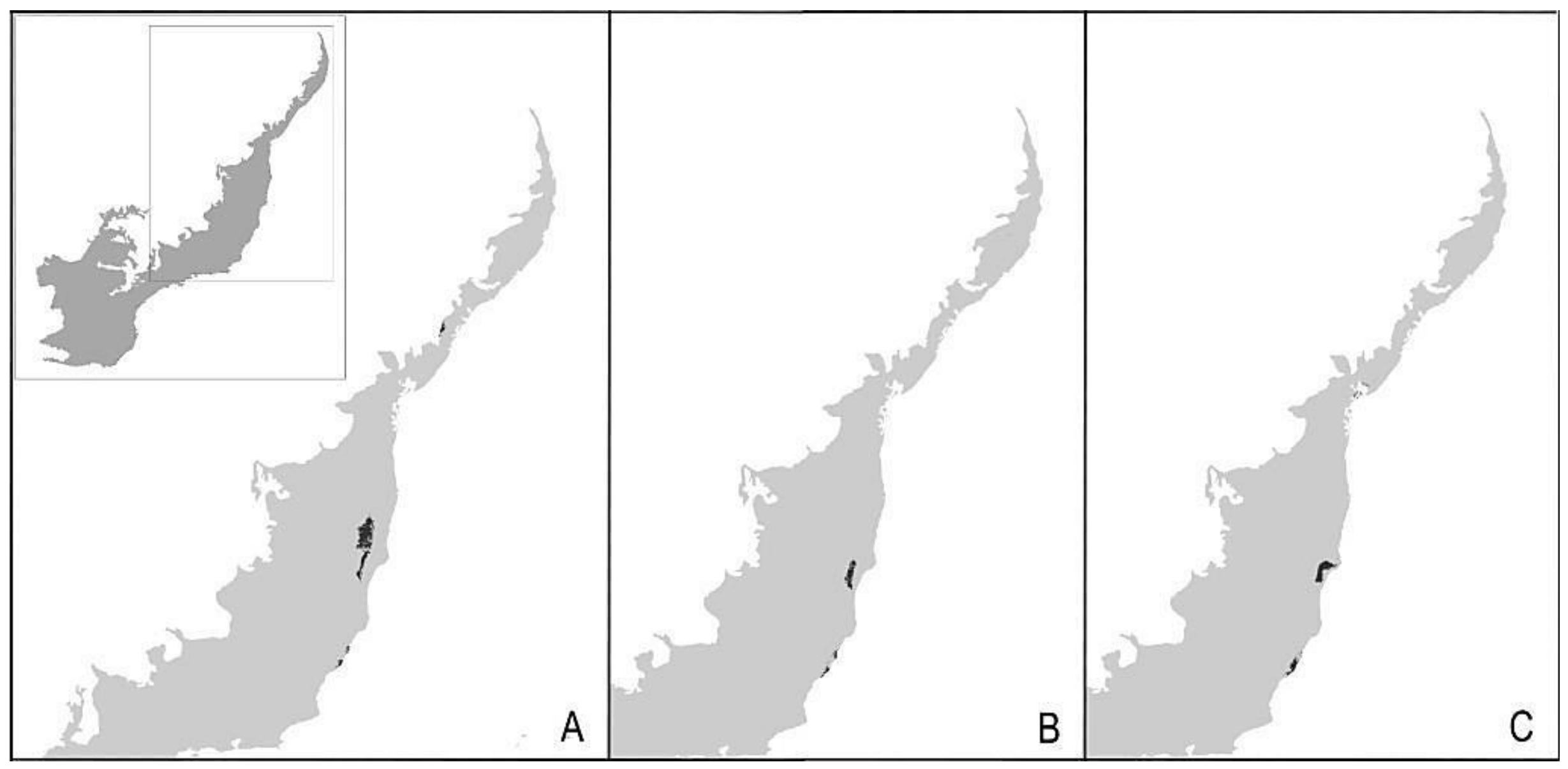

FIGURE S14: Estimated current (A) and future habitat suitability in the B2a (B) and A2a (C) climatic scenario for the year 2080 in the Brazilian Atlantic forest for Melanophryniscus setiba. Modeled distributions shown in black. 


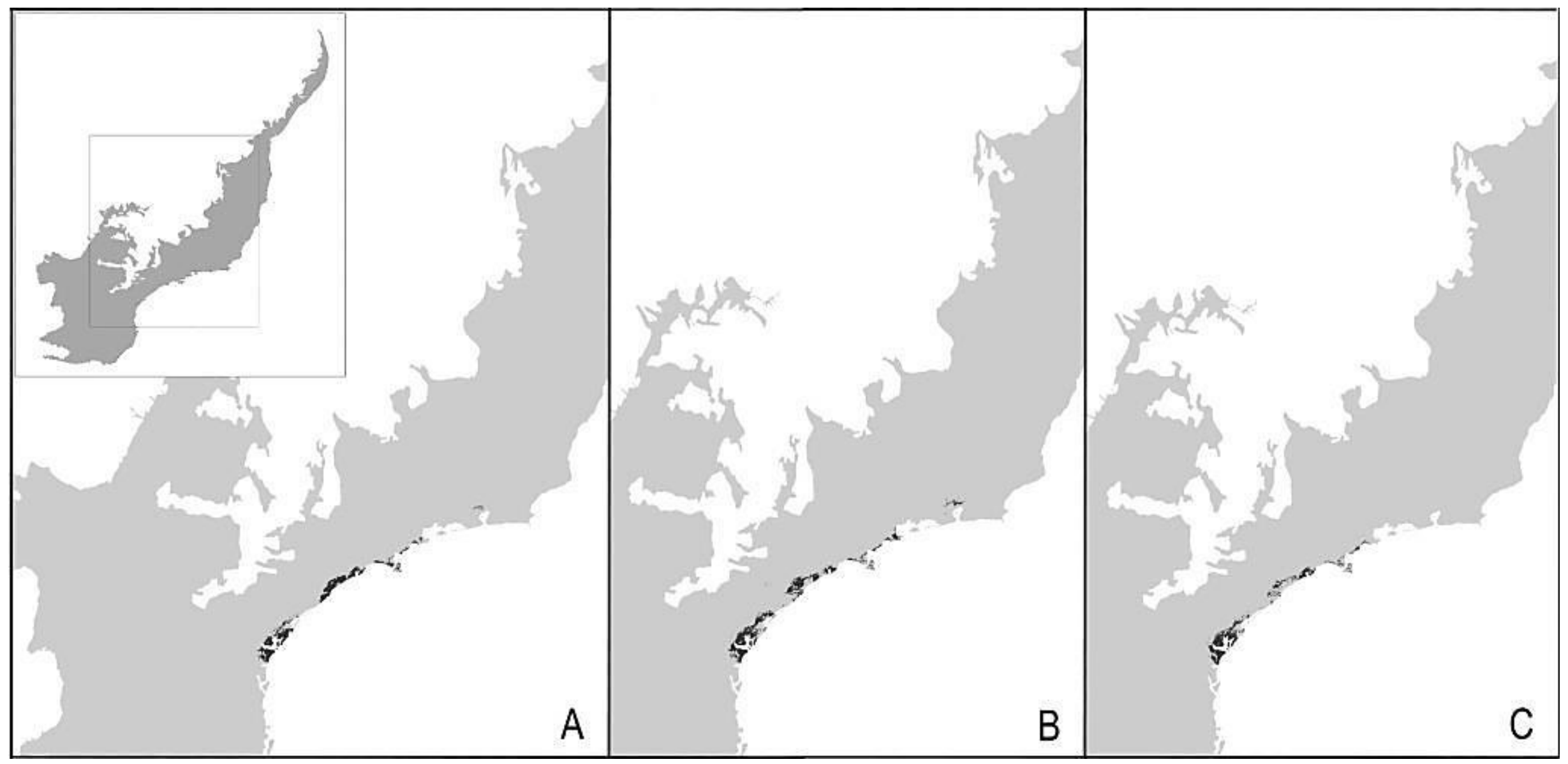

FIGURE S15: Estimated current (A) and future habitat suitability in the B2a (B) and A2a (C) climatic scenario for the year 2080 in the Brazilian Atlantic forest for Physalaemus atlanticus. Modeled distributions shown in black. 


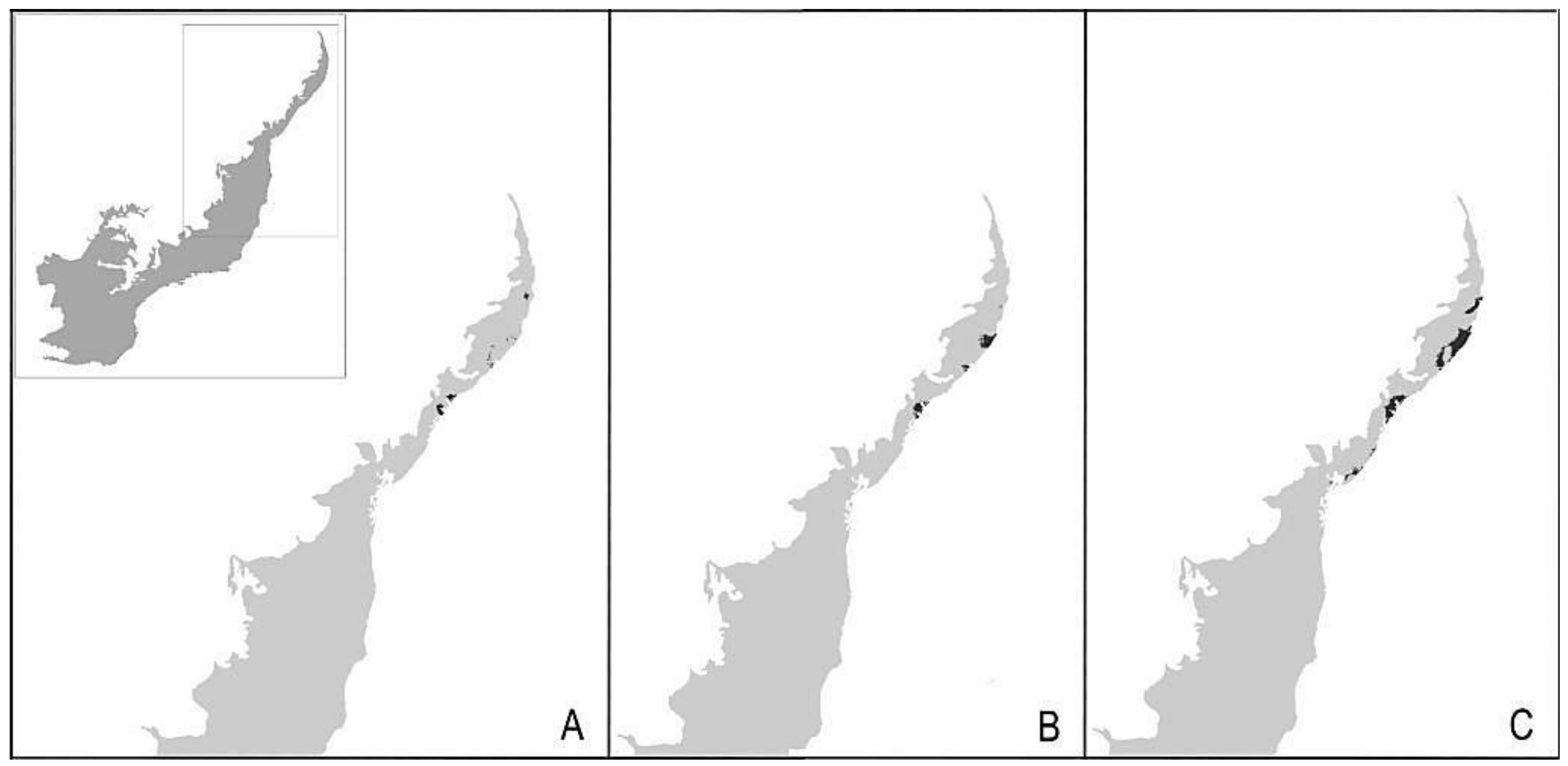

FIGURE S16: Estimated current (A) and future habitat suitability in the B2a (B) and A2a (C) climatic scenario for the year 2080 in the Brazilian Atlantic forest for Phyllodytes punctatus. Modeled distributions shown in black. 


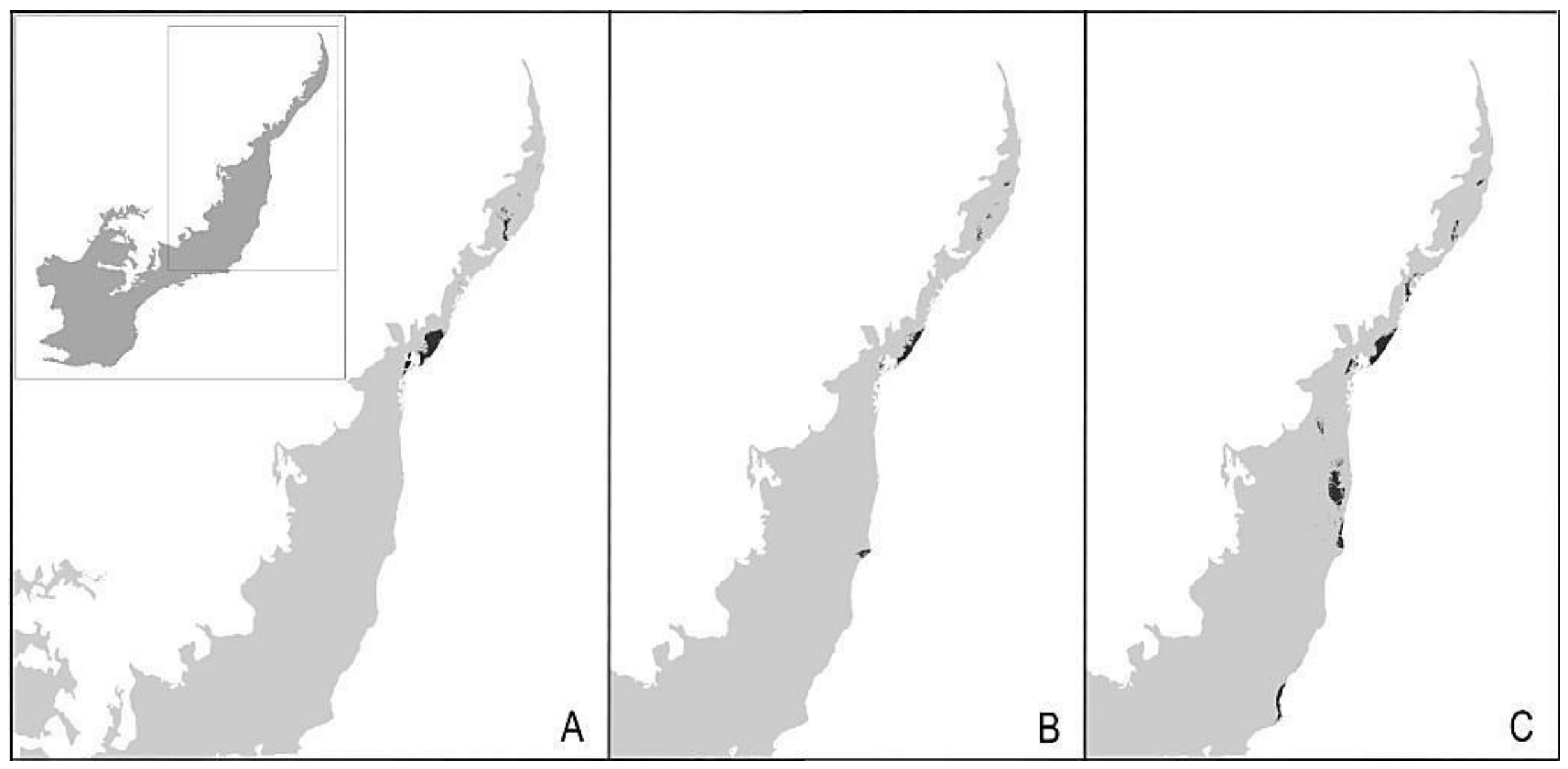

FIGURE S17: Estimated current (A) and future habitat suitability in the B2a (B) and A2a (C) climatic scenario for the year 2080 in the Brazilian Atlantic forest for Scinax cretatus. Modeled distributions shown in black. 


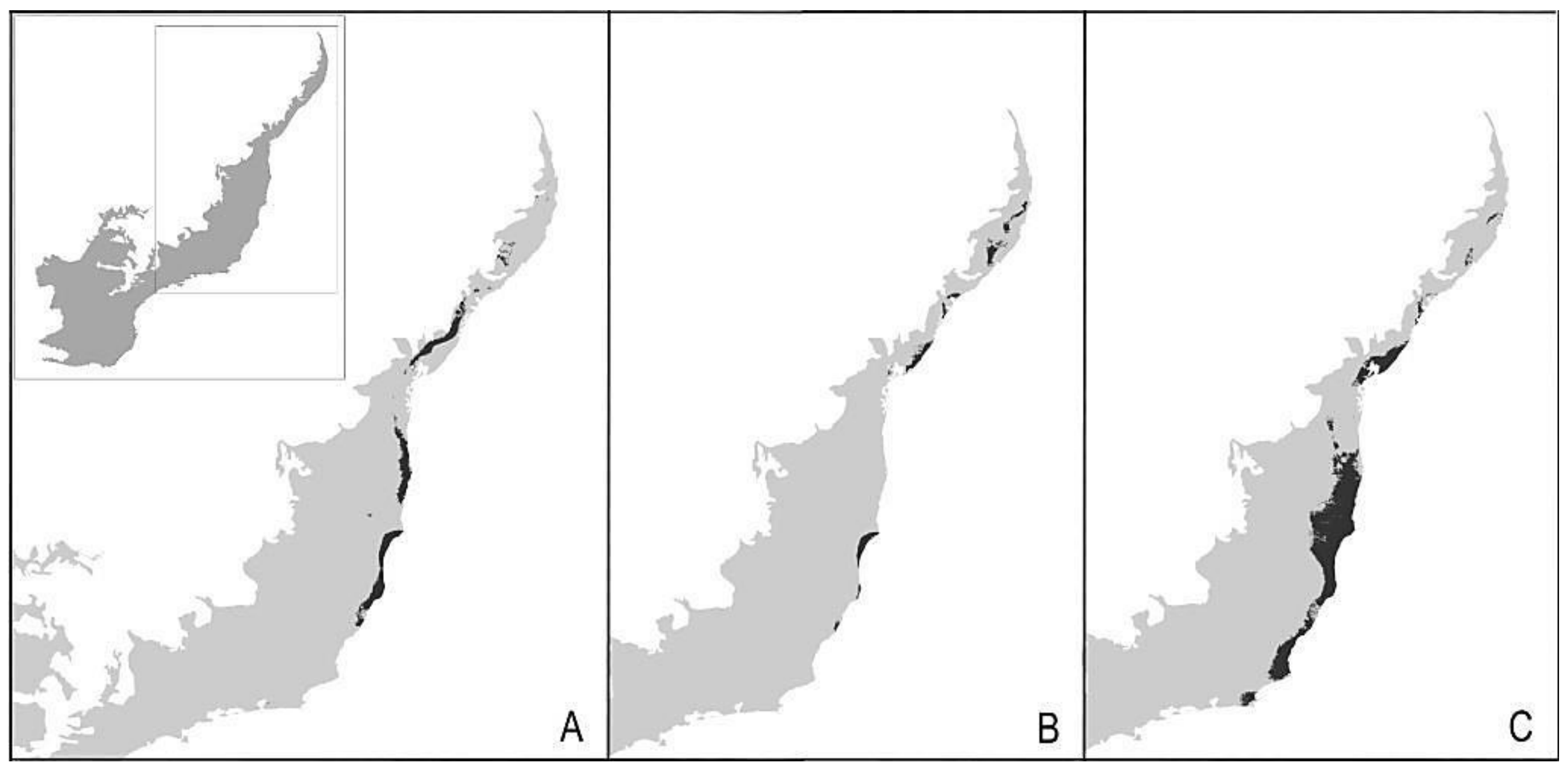

FIGURE S18: Estimated current (A) and future habitat suitability in the B2a (B) and A2a (C) climatic scenario for the year 2080 in the Brazilian Atlantic forest for Sphaenorhynchus palustris. Modeled distributions shown in black. 


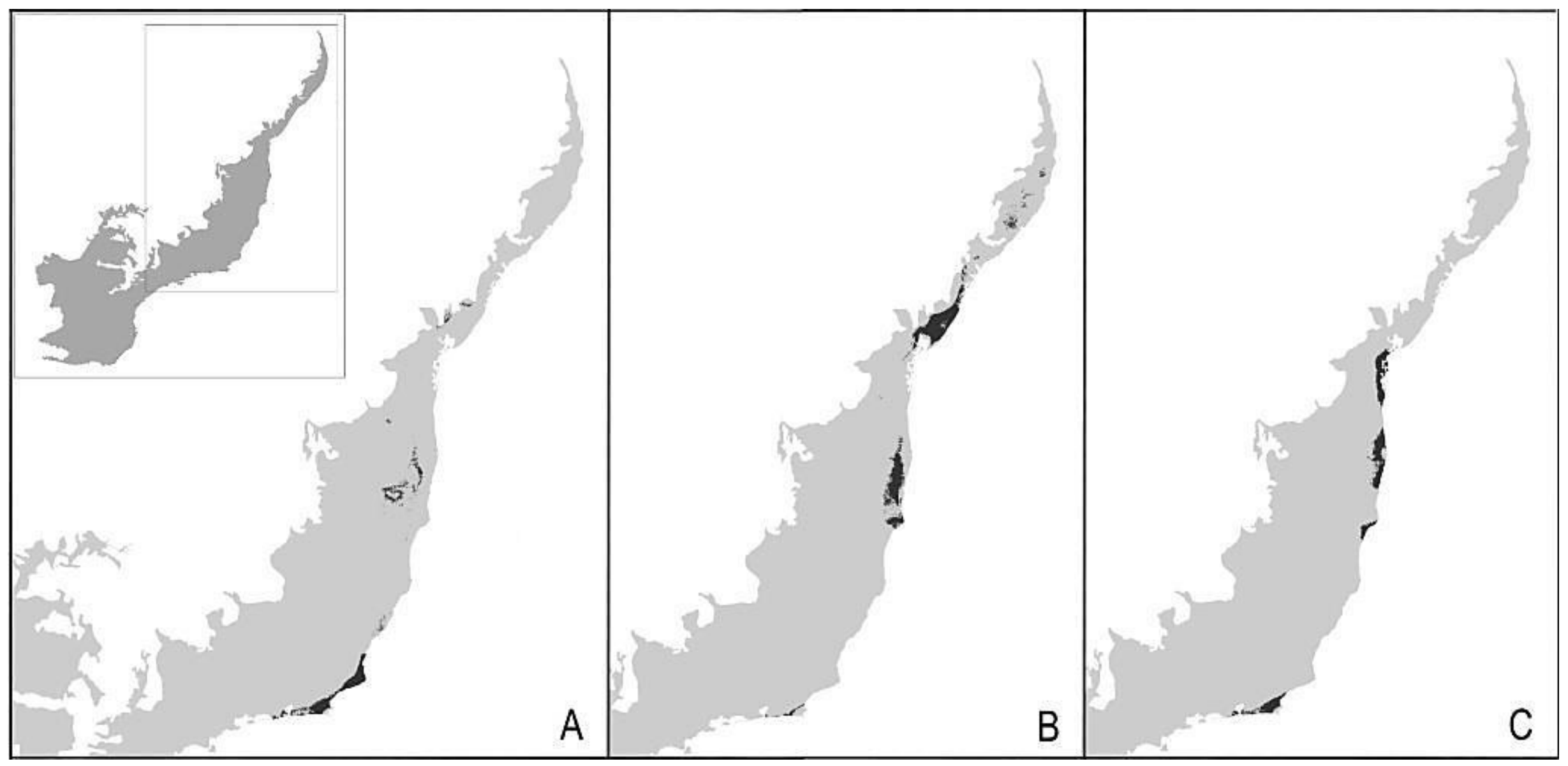

FIGURE S19: Estimated current (A) and future habitat suitability in the B2a (B) and A2a (C) climatic scenario for the year 2080 in the Brazilian Atlantic forest for Xenohyla truncata. Modeled distributions shown in black. 